\title{
Metodología miceps para control estadístico de procesos: caso aplicado al proceso de producción de vidrio templado
}

\author{
Miceps methodology for statistical process control: \\ a case study applied in production process of tempered glass
}

\author{
Miguel Angel Ortíz Barrios ${ }^{1}$, Heriberto Alexander Felizzola Jiménez ${ }^{2}$ \\ ${ }^{1}$ Ingeniero Industrial. Especialista en Gerencia de Producción y Operaciones. Docente Investigador de la Universidad de la Costa CUC. \\ Barranquilla, Colombia. Grupo de investigación PRODUCOM. mortiz1@cuc.edu.co. \\ ${ }^{2}$ Ingeniero Industrial. Magister en Ingeniería Industrial. Docente Investigador de la Universidad de la Salle. Bogotá, D. C., Colombia. \\ Email:mortiz1@cuc.edu.co
}

Recibido: 22/04/14 / Aceptado: 3/06/14

Citar como: D.C. Manco, E.E.Rojas, B.A. Gomez, “Miceps methodology for statistical process Control: a case study applied in production Process of tempered glass ", Prospect, Vol 12, N², 73-81, 2014.

\section{RESUMEN}

El Control Estadístico de procesos es considerado como una herramienta de la Gestión de la Calidad orientada a la mejora de procesos y productos. Su aplicación exitosa en la industria manufacturera y de servicios ha representado beneficios a nivel financiero y de mercado. Para ello, es importante garantizar una efectiva articulación entre las perspectivas del cliente y la compañía. Por tal motivo, el presente estudio tiene como objetivo el desarrollo de un enfoque basado en la metodología MICEPS (Methodology for integrating customer expectations and production systems) que ayuda a las compañías a traducir las expectativas del cliente en sistemas efectivos de control de calidad. Primero, se efectúa un análisis de proceso y luego se aplica el enfoque MICEPS para el establecimiento de puntos de monitoreo y control basados en el cliente. Un caso de estudio de la industria de vidrio templado ha sido empleado para explorar la efectividad del enfoque propuesto.

Palabras clave: Gestión de la calidad; Control Estadístico de procesos; Críticos de satisfacción del cliente; MICEPS; Vidrio templado.

\begin{abstract}
Statistical Process Control is regarded as a Quality Management tool for the improvement of processes and products. Its successful implementation on manufacturing and service industries has provided financial and market benefits. Therefore, it is very important to guarantee an efficacious link between customer expectations and companies. This study aims to develop an approach based on MICEPS (Methodology for integrating customer expectations and production systems) to help companies design effective systems of quality control. First, a process analysis is carried out and then MICEPS is applied to establish monitoring and control points with customer focus. A case study from tempered glass industry is used to explore the effectiveness of the proposed approach.
\end{abstract}

Key words: Quality management; Statistical Process Control; Critical to customer satisfaction; MICEPS; Tempered glass.

\section{INTRODUCCIÓN}

El Control Estadístico de Procesos (CEP) y la Gestión de Calidad son dos estrategias adscritas a la mejora de productos y procesos. En los últimos años, muchas compañías de clase mundial las han adoptado obteniendo excelentes resultados a nivel financiero y de mercado [1]. El principal beneficio derivado de la implementación de estos programas es la reducción de la variabilidad [2] a través de la creación de un sistema en el cual la organización es capaz de recolectar, analizar y desplegar datos de manera consistente [3], lo que permite construir una estructura que garantiza el continuo mejoramiento de la calidad en soporte con la toma de decisiones basada en el cliente. 
Las necesidades y expectativas de los clientes son el principal insumo de toda compañía. Con ellas, se diseñan los sistemas de calidad y producción que hacen posible la fabricación de los bienes y servicios que buscarán satisfacer el mercado. Por ello, El enfoque actual de la calidad señala que es necesario que las compañías concentren sus esfuerzos y recursos en el perfeccionamiento continuo de sus operaciones para lograr la satisfacción total de sus clientes [4].

El éxito en la implementación del SPC en un empresa de manufactura o servicios, está condicionado al manejo que se ejerce sobre una serie de aspectos claves como: el compromiso de la dirección con la mejora continua, la alineación con la estrategia de la organización, la alineación con los críticos de satisfacción del cliente (CTS), la experticia de los equipos de trabajo en manejo de técnicas estadísticas aplicadas a la gestión y mejora de la calidad, la identificación de procesos claves, el conocimiento del sistema en estudio y la inversión en recursos para la ejecución de los procesos de control estadístico de la calidad [5]. Cada uno de estos elementos constituye un aporte fundamental a la implementación del SPC, por lo cual se hace posible abordar aspectos que van más allá de la simple elaboración e interpretación de gráficos. De esta manera, la organización es capaz de diseñar e implementar acciones efectivas que mejoren la estabilidad y variabilidad de sus procesos y por ende, la satisfacción de sus clientes.

Este artículo está organizado de la siguiente manera: Primero, se presenta una revisión literaria del tema desarrollado en el estudio. Posteriormente, se presenta una metodología para la integración efectiva de los requerimientos del cliente con los sistemas de producción y calidad de una compañía. En tercer lugar, se desarrolla un caso de estudio real para demostrar la factibilidad de la metodología propuesta, y finalmente, se enuncian las conclusiones derivadas del estudio.

\section{MODELOS Y METODOLOGIAS PARA LA IMPLEMENTACIÓN DE SISTEMAS DE CONTROL ESTADISTICO DE PROCESOS}

Basados en la necesidad de abordar los aspectos metodológicos concernientes a la implementación de sistemas de control estadístico de procesos, algunos expertos en el tema han propuesto modelos y metodologías que sirven como guía a las organizaciones en el propósito de determinar las actividades a desarrollar y los factores claves que deben abordarse, para que el control estadístico se gestione de una forma adecuada y cumpla su objetivo: garantizar que los procesos sean estables, con baja dispersión, desempeño medible en el tiempo y alta capacidad en el cumplimiento de especificaciones.

(Oakland, 2003) presenta una metodología enfocada a la realización de procesos de mejora, iniciando con la selec- ción y análisis de los procesos a intervenir, luego la definición de los requerimientos de información y datos, su recolección y análisis, seguidamente el desarrollo de las mejoras y por último, el establecimiento de procesos de monitoreo y control para garantizar su correcto funcionamiento. Si bien el modelo propuesto posee una gran utilidad para el desarrollo de procesos de mejora, no brinda una guía suficiente para abordar aspectos relacionados con: la identificación de los CTS, la priorización de procesos a partir de los CTS y la estrategia de la organización, la validación de los sistemas de medición y la ejecución de herramientas claves como el diseño de experimentos y el diseño de parámetros como soporte a los procesos de mejora [6].

Antony and Taner (2003) expone un modelo enfocado sobre áreas claves en el desarrollo de un sistema de control de calidad, tales como: aspectos relacionados con la gestión y la dirección, el desarrollo de competencias en ingeniería de procesos, formación en técnicas estadísticas y el desarrollo de equipos de trabajo. Sin embargo, una de las falencias más notables en el modelo propuesto, es la falta de una metodología o enfoque que precise la integración de la voz del cliente con el proceso de monitoreo y el control de variables críticas [7].

Salah et al. (2010) propone la integración de la metodología Seis Sigma y el enfoque lean para guiar a las compañías hacia la satisfacción continua de las necesidades sus clientes. Para ello, el modelo incluye las prácticas lean dentro de la fase de mejora del ciclo DMAIC; más sin embargo no muestra una aplicación real que valide su alcance y beneficios [8].

Behbahani et al. (2012) desarrollan un modelo para la implantación de SPC fundamentado en un estudio de casos de éxito desarrollados en diferentes empresas, por lo que se garantiza que los factores abordados han sido considerados con base en hechos reales y no en conjeturas [9].

(Kulahci, 2013) presenta la implementación del control estadístico de procesos multivariados en diferentes sectores de la industria, aplicando cartas de control multivariadas asociadas a la utilización de tecnologías eficientes en el análisis de datos y las cuales permiten optimizar los procesos de análisis y tomas de decisiones [10].

Haciendo un análisis en conjunto de todos los modelos estudiados, se puede observar que todos se enfocan principalmente en cuatro elementos: la preparación de equipos de trabajo, la selección y análisis de procesos, la selección de cartas de control y el tratamiento de problemas basado en control estadístico de procesos. Si bien cada modelo propone elementos de suma importancia para la implementación del SPC, también se puede apreciar que casi 
todas carecen de un enfoque definido para vincular la voz del cliente dentro de la identificación de procesos y variables críticas, lo cual es determinante dentro de un mercado donde las necesidades de los clientes son cada vez más cambiantes y donde éstos son cada vez menos tolerantes a los errores.

\section{METODOLOGIA PROPUESTA}

La metodología diseñada para la integración efectiva entre los requerimientos del cliente y los sistemas de producción y calidad de una compañía (MICEPS), consta principalmente de seis fases: Análisis de proceso, Identificación de CTS, Construcción de la matriz de influencias variable - proceso, Construcción de la matriz de influencias CTS - Variable de proceso, Análisis del modo y efecto de falla en el proceso y Diseño de puntos de inspección control en el proceso. Para cada fase se enlistan los objetivos propuestos, se hace una descripción breve de las actividades que deben llevarse a cabo y se definen los resultados que deben ser alcanzados al final de su aplicación.

\section{Fase 1: Análisis de proceso}

Objetivo: Identificar las diferentes etapas del proceso y establecer las interacciones existentes entre las diferentes etapas del proceso.

Descripción de actividades: En esta fase, se procede en primer lugar al diseño y aplicación de instrumentos de recolección de información acordes con el proceso en estudio (notas, fotografías, formatos). Seguidamente, se propone la realización de un diagrama de procesos que permita la identificación de las etapas del proceso e interacciones asociadas. Por último, a través de un diagrama SIPOC, se identifican los inputs y outputs correspondientes a cada etapa del proceso.

Resultados: Flujograma de proceso, fotografías, notas y SIPOC por cada etapa del proceso.

\section{Fase 2: Identificación de CTS}

Objetivo: Identificar las características de calidad que son críticas para el cliente.

Descripción de actividades: Luego de haber realizado el análisis de proceso, se continúa con la identificación de las expectativas del cliente las cuales posteriormente se clasifican en CTD (Crítico de entrega), CTQ (Crítico de calidad) y CTC (Crítico de costo) según sea su naturaleza.

Resultados: Críticos de satisfacción del cliente.
Fase 3. Construcción de la matriz de influencias Variable - proceso

Objetivo: Identificar los subprocesos con mayor presencia de variables de proceso y establecer las variables de proceso con mayor presencia a lo largo de la línea de producción.

Descripción de actividades: En primera medida, se identifican las variables de proceso presentes en cada etapa de la línea de producción. Por último se construye una matriz de influencias Variable - proceso donde se busca establecer las variables de proceso con mayor presencia a lo largo de la línea de producción. Para ello, se procede al enlistado, en primera instancia, de todos los subprocesos de la línea de fabricación en estudio así como las variables de proceso previamente identificadas durante el trabajo de campo. Posteriormente, se examina la relación entre cada subproceso involucrado y una determinada variable de proceso, y por último, se totaliza el número de variables presentes por subproceso así como el número de subprocesos en el que cada variable está presente

Resultados: Variables de proceso con mayor presencia a lo largo de la línea de producción y subproceso con mayor presencia de variables.

Fase 4. Construcción de la matriz de influencias CTS - Variables de proceso

Objetivo: Definir las variables de proceso que son críticas para el cumplimiento de los requerimientos del cliente.

Descripción de actividades: En esta etapa, se construye la matriz de influencias CTS - Variables de proceso, una adaptación del diagrama causa-efecto a fin de identificar las variables de proceso que son críticas para el cumplimiento de los requerimientos del cliente. Para ello, se procede a especificar el grado de incidencia de la variable de proceso en el cumplimiento de cada CTS de acuerdo con la siguiente escala: No hay relación (0), relación extremadamente débil (1), relación débil (2), relación moderada (3), relación fuerte (4) y relación extremadamente fuerte (5). Por último, se efectúa una sumatoria de los puntajes otorgados a cada variable de proceso.

Resultados: Variables de proceso críticas para el cumplimiento de los requerimientos del cliente.

\section{Fase 5. Análisis del modo y efecto de falla en el proceso (AMEF)}

Objetivos: Diseñar planes enfocados a la mitigación del impacto negativo de los modos de falla en el nivel de satisfacción del cliente. 
Descripción de actividades: En primera instancia, se enlistan los modos potenciales de falla para cada una de las variables de proceso identificada como crítica en la fase anterior. Luego, se identifican los posibles efectos de cada modo de falla dada su ocurrencia. Seguidamente, se determinan las posibles causas de aparición de los modos de falla en estudio y se describen los controles existentes para su detección en el proceso. Como paso siguiente, se evalúa la severidad, ocurrencia y detección de los modos de falla de acuerdo a la escala de calificación mostrada en las tablas 1, 2 y 3 [11] para posteriormente calcular el índice RPN. Por último, se establecen los modos de falla críticos y se diseñan planes de acción para su mitigación.

Resultados: Planes de mitigación para los modos de falla críticos.

Fase 6. Diseño de puntos de inspección y control en el proceso

Objetivo: Definir los puntos de control a implementar en el sistema productivo para monitorear y controlar las variables críticas de proceso.

Descripción de actividades: En la fase de diseño de puntos de inspección y control, se hace necesaria la creación de métrico (s) de desempeño para cada modo de falla crítico. Como siguiente paso, se procede con la definición de los puntos de inspección y control en la línea de producción estableciendo su localización en los subprocesos que pre- sentan mayor frecuencia de aparición de defectos. Seguidamente, se asignan las actividades de inspección para cada punto de control instalado. Como siguiente medida, se diseña el método de inspección y se efectúa un estudio R\&r a fin de determinar la confiabilidad de las mediciones y por ende, la confiabilidad de las decisiones consecuentes. Por último, se diseña un plan de muestreo acorde al comportamiento de la producción que permita el monitoreo de los métricos de desempeño a través de la instalación de cartas de control y la utilizaciónn de métodos de análisis estadístico correspondientes.

Resultados: Puntos de inspección y control de CTS en el proceso.

\section{CASO DE ESTUDIO}

El caso de estudio propone la aplicación de la metodología MICEPS en la línea de fabricación de vidrio templado de una empresa colombiana la cual, debido a las altas exigencias en materia de calidad y tiempo de entrega a la que se ve sometida por parte de las industrias del sector de construcción, busca la implantación de mecanismos que disminuyan el índice actual de sobrecostos por materiales defectuosos y/o retrasos presentados en la entrega de sus productos; de tal modo, que se disminuya el riesgo de demandas por incumplimiento, cancelaciones contractuales y pérdida de relaciones comerciales de gran importancia para su sostenimiento financiero en el futuro.

Tabla 1. Escala de calificación para AMEF.

Table 1. Qualification scale for AMEF.

\begin{tabular}{|c|c|c|c|}
\hline Puntaje & Severidad del Efecto & $\begin{array}{c}\text { Probabilidad de } \\
\text { ocurrencia }\end{array}$ & Detección \\
\hline 10 & Peligroso sin aviso & \multirow{2}{*}{$\begin{array}{l}\text { Muy Alta: } \\
\text { Fallo casi inevitable }\end{array}$} & Casi imposible \\
\hline 9 & Peligroso con aviso & & Muy remota detección \\
\hline 8 & Pérdida de función primaria & \multirow{2}{*}{$\begin{array}{c}\text { Alta: } \\
\text { Fallos frecuentes }\end{array}$} & Remota detección \\
\hline 7 & Rendimiento disminuido en función primaria & & Muy baja detección \\
\hline 6 & Pérdida de función secundaria & \multirow{3}{*}{$\begin{array}{l}\text { Moderada: } \\
\text { Fallos ocasionales }\end{array}$} & Baja detección \\
\hline 5 & Rendimiento disminuido en función secundaria & & Moderada detección \\
\hline 4 & $\begin{array}{c}\text { Defecto minúsculo notado por la mayoría } \\
\text { de los clientes }\end{array}$ & & $\begin{array}{l}\text { Posibilidad moderadamente alta } \\
\text { de detección }\end{array}$ \\
\hline 3 & Defecto minúsculo notado por algunos clientes & \multirow{2}{*}{$\begin{array}{c}\text { Baja: } \\
\text { Fallas aisladas }\end{array}$} & Alta detección \\
\hline 2 & $\begin{array}{l}\text { Defecto minúsculo notado por clientes muy } \\
\text { detallistas }\end{array}$ & & Muy alta detección \\
\hline 1 & Ninguno & $\begin{array}{c}\text { Remota: } \\
\text { Fallo poco probable }\end{array}$ & Detección casi segura \\
\hline
\end{tabular}


Tabla 2. Criterios para la calificación de la probabilidad de ocurrencia de las causas potenciales de falla. Table 2. Criteria for the ranking of occurrence probability in potential causes of failure.

\begin{tabular}{|c|c|c|}
\hline Probabilidad de ocurrencia de la causa que provoca la falla & Tasa de falla & Puntuación \\
\hline \multirow{2}{*}{ Muy alta: fallo casi inevitable } & $>100$ por cada 1000 láminas de vidrio & 10 \\
\cline { 2 - 3 } & 50 por cada 1000 láminas de vidrio & 9 \\
\hline \multirow{2}{*}{ Alta: fallos frecuentes } & 20 por cada 1000 láminas de vidrio & 8 \\
\cline { 2 - 3 } & 10 por cada 1000 láminas de vidrio & 7 \\
\hline \multirow{2}{*}{ Moderada: fallos ocasionales } & 5 por cada 1000 láminas de vidrio & 5 \\
\cline { 2 - 3 } & 2 por cada 1000 láminas de vidrio & 3 \\
\cline { 2 - 3 } & 1 por cada 1000 láminas de vidrio & 2 \\
\hline \multirow{2}{*}{ Baja: fallas aisladas } & 0.5 por cada 1000 láminas de vidrio & 1 \\
\hline
\end{tabular}

Tabla 3. Criterios para la calificación de la probabilidad de detección de fallas por parte de los controles.

Table 3. Criteria for the ranking of failure detection probability by controls.

\begin{tabular}{|c|c|c|}
\hline Detección & Probabilidad de detección de la falla (\%) & Puntaje \\
\hline Casi imposible & Menor a 90\% & 10 \\
\hline Muy remota detección & $90 \%$ & 9 \\
\hline Remota detección & \multirow{2}{*}{$98 \%$} & 8 \\
\hline Muy baja detección & & 7 \\
\hline Baja detección & \multirow{2}{*}{$99.7 \%$} & 6 \\
\hline Moderada detección & & 5 \\
\hline Posibilidad moderadamente alta de detección & \multirow{2}{*}{9} & 4 \\
\hline Alta detección & & 3 \\
\hline Muy alta detección & $99.99 \%$ & 2 \\
\hline Detección casi segura & & 1 \\
\hline
\end{tabular}

El presente estudio tiene un gran impacto sobre el funcionamiento de la línea de producción mencionada dada la poca conciencia de calidad que la compañía refleja en cada una de sus jerarquías organizacionales. Por otro lado, la empresa no cuenta con registros históricos que posibiliten la identificación de patrones de comportamiento estadístico y de variación relacionados con las variables críticas del proceso de fabricación; hecho que redunda en la generación de producto defectuoso, el aumento de costos de no calidad, retrasos en la entrega del producto y por ende, la disminución del nivel de satisfacción de los clientes.

\subsection{Primera fase: Análisis de proceso}

Para esta primera fase, se diseñó y aplicó un formato de recolección de información que permitió definir los subprocesos componentes de la línea de producción de vidrio templado así como las posibles rutas que pudiera seguir el producto dada sus características; hecho a través del cual se pudo establecer la existencia de un sistema productivo tipo job-shop (ver figura 1).

Por otro lado, se definieron las entradas y salidas para cada uno de los subprocesos de la línea de producción y se construyeron los diagramas SIPOC (Proveedores, Entradas, Proceso, Salidas y Clientes) respectivos para la identificación de todos los elementos relevantes en el proceso (ver tabla 4). 
Figura 1. Diagrama de flujo para la línea de producción de vidrio templado.

Figure 1. Flow diagram for glass tempered production line.

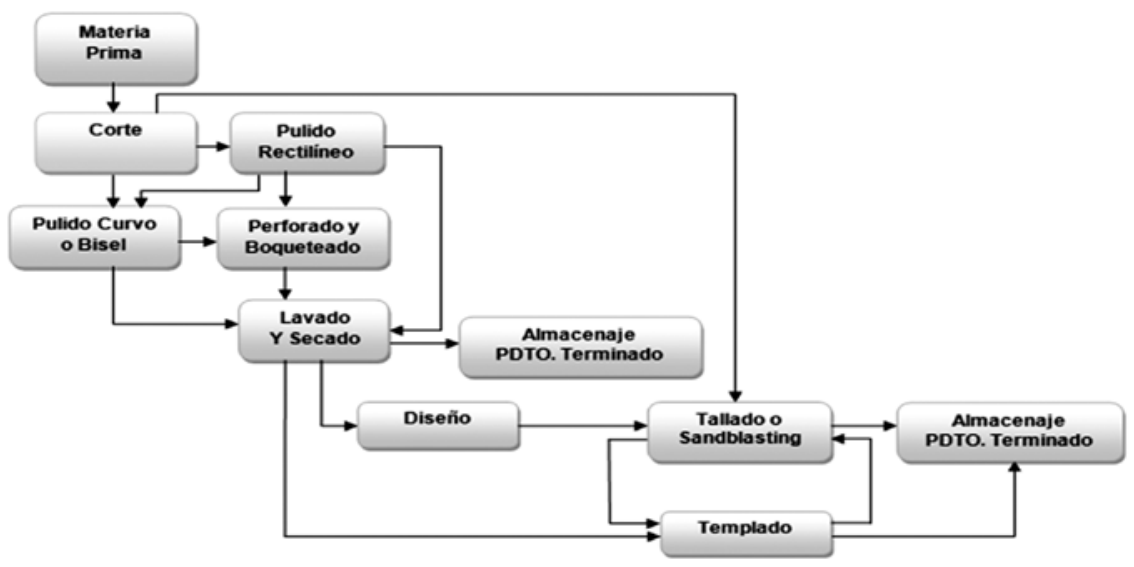

Tabla 4. Diagrama SIPOC para el subproceso de corte de vidrio.

Table 4. SIPOC diagram for glass cutting subprocess.

\begin{tabular}{|c|c|c|c|c|}
\hline PROVEEDOR & ENTRADAS & PROCESO & SALIDAS & CLIENTE \\
\hline \multirow{2}{*}{ Producción } & $\begin{array}{l}\text { Formato de } \\
\text { producción }\end{array}$ & \multirow{14}{*}{ CORTE } & \multirow{2}{*}{$\begin{array}{l}\text { Módulos de Vidrio cortadas } \\
\text { a la medida con etiqueta con } \\
\text { código de prioridad }\end{array}$} & \multirow{2}{*}{$\begin{array}{l}\text { Pulido Rectilíneo } \\
\text { Tallado }\end{array}$} \\
\hline & $\begin{array}{l}\text { Etiqueta con código de } \\
\text { prioridad }\end{array}$ & & & \\
\hline \multirow{9}{*}{$\begin{array}{c}\text { Almacén de Insumos y } \\
\text { Herramientas }\end{array}$} & Módulo de vidrio & & \multirow{5}{*}{$\begin{array}{l}\text { Módulos de vidrio con } \\
\text { bordes curvos con etiqueta } \\
\text { con código de prioridad }\end{array}$} & \multirow{5}{*}{ Pulido Curvo } \\
\hline & Corta vidrios & & & \\
\hline & Pinzas de presión & & & \\
\hline & Reglas, escuadras & & & \\
\hline & Compás & & & \\
\hline & Flexómetro & & $\begin{array}{l}\text { Registro de } \\
\text { producción }\end{array}$ & Producción \\
\hline & Mesa de Corte & & \multirow{3}{*}{$\begin{array}{l}\text { Número de horas de paro } \\
\text { por avería en máquina }\end{array}$} & \multirow{3}{*}{ Mantenimiento } \\
\hline & Plantilla & & & \\
\hline & ACPM - Refrigerante & & & \\
\hline Mantenimiento & $\begin{array}{l}\text { Plan de mantenimiento } \\
\text { preventivo y/o correctivo }\end{array}$ & & Residuos de vidrio & \\
\hline \multirow{2}{*}{$\begin{array}{l}\text { Salud Ocupacional y } \\
\text { Recursos Humanos }\end{array}$} & EPP & & & \\
\hline & Manual de funciones & & & \\
\hline
\end{tabular}

\subsection{Segunda fase: Identificación de CTS}

En la segunda fase, se procedió con la identificación de los CTS (Críticos de satisfacción del cliente). Para el caso de estudio, se utilizaron los juicios emitidos por expertos representantes de los diferentes departamentos de la com- pañía, logrando identificar 4 clientes principales (ver tabla 5): Cliente externo, Dpto. de Producción, Dpto. Control de calidad y Contratistas. Posteriormente, se clasificaron los CTS (ver tabla 5) dentro de la tipología CTQ (Crítico de calidad), CMTD (Crítico de entrega) y CTC (Crítico de costo). 
4.3 Tercera fase: Construcción de la matriz de influencias variable-proceso

En esta fase, se construyó una MATRIZ DE INFLUENCIAS VARIABLE - PROCESO, herramienta que permitió establecer la existencia de interrelaciones entre cada uno de los procesos de la compañía y las variables de proceso involucradas.

Seguidamente, se procedió a identificar los subprocesos con mayor presencia de variables y las variables con mayor presencia a lo largo de la línea de producción. Para el caso en estudio se determinó que:

- El subproceso de Templado presenta el mayor número de variables con 22, seguido por el subproceso de perforado con 12.

- El subproceso de Templado cuenta con 16 variables propias de las 22 que posee.

- Las variables de transporte y descargue de material, interpretación de la orden de producción y ubicación de materiales en zona de almacenamiento se presentan en los 9 subprocesos objeto de estudio.

\subsection{Cuarta fase: Construcción de la matriz de influen- cias CTS-variables de proceso}

La siguiente etapa de esta metodología involucra la construcción de la MATRIZ DE INFLUENCIAS CTS - Variables de proceso (ver tabla 6), estación en la cual se busca determinar la incidencia de las variables de proceso previamente identificadas en el logro de los distintos críticos de satisfacción anteriormente establecidos.

La aplicación de esta matriz de influencias generó como resultado, la creación de un ranking de variables en el que se evidencian las variables de proceso que será necesario monitorear para garantizar la satisfacción continua del cliente. Para el caso de estudio, se determinó que las variables USO DEL FLEXOMETRO, TÉCNICA DE AVELLANADO, UNIFORMIDAD DEL BORDE DE VIDRIO y UNIFORMIDAD DEL BORDE EN EL AGUJERO son críticas en la línea de producción y por ende, deben ser objeto de monitoreo.

\subsection{Quinta fase: Análisis del modo y efecto de falla en el proceso}

Para la quinta fase, se diseñó un AMEF que permitió detectar los modos de falla críticos en la línea de producción (ver tabla 7). Para cada modo de falla crítico, se establecieron planes de acción con el fin de garantizar el cumplimiento de los CTS asociados a su campo de acción.

\subsection{Sexta fase: Diseño de puntos de control en el proceso}

En la fase final, se procedió al diseño de métricos para el monitoreo y control de los efectos de falla asociados y previamente identificados a través del AMEF (ver tabla 8). Luego, se establecieron puntos de control en la línea de producción sobre los subprocesos de pulido rectilíneo y perforado, los cuales presentaron una mayor frecuencia de aparición de defectos asociados a los modos de falla previamente definidos. Seguidamente, se asignaron las actividades de inspección para cada punto de control instalado (ver tabla 9) y se realizó una prueba de reproducibilidad y repetibilidad R\&r que arrojó un \%GageR\&r del $22 \%$ por lo cual se concluye que el sistema de medición se considera ACEPTABLE. Finalmente, se diseñó un plan de muestreo según tamaño de lote con $S^{2}=5$ y $\alpha=95 \%$ en complemento con la implantación de cartas de control X y $\mathrm{R}$ para el monitoreo de las dimensiones ancho y longitud de los módulos de vidrio y la carta U para el monitoreo de defectos por lote de inspección.

Tabla 5. Procesos de la compañía relacionados con el cumplimiento de los CTS del cliente externo.

Table 5. Company processes related to the fulfillment of external customer CTS.

\begin{tabular}{|c|c|c|c|}
\hline CLIENTE & & CTS & $\begin{array}{l}\text { PROCESOS RELACIONADOS CON EL } \\
\text { CUMPLIMIENTO DEL CTS }\end{array}$ \\
\hline \multirow{9}{*}{$\begin{array}{l}\text { CLIENTE } \\
\text { EXTERNO }\end{array}$} & \multirow{3}{*}{ CTD } & Tamaño correcto de pedido. & Despacho, Producción \\
\hline & & Pedido coherente con la solicitud del cliente. & Despacho \\
\hline & & Entrega a tiempo del producto. & Despacho, Producción y Ventas \\
\hline & \multirow{4}{*}{ CTQ } & Producto en color (es) correcto (s) & Ventas, Producción (Subproceso de Corte) \\
\hline & & Producto sin defectos. & Producción \\
\hline & & Producto correctamente instalado. & Instalación \\
\hline & & Producto en dimensiones correctas. & $\begin{array}{c}\text { Mantenimiento, Ventas, Producción y } \\
\text { Proveedor (suministro de equipos calibrados) }\end{array}$ \\
\hline & \multirow{2}{*}{ СтC } & Precio atractivo del producto. & Ventas \\
\hline & & Precio atractivo de la instalación. & Ventas \\
\hline
\end{tabular}


Tabla 6. Matriz de influencias CTS - Variable de proceso.

Table 6. CTS - Process variable influence matrix.

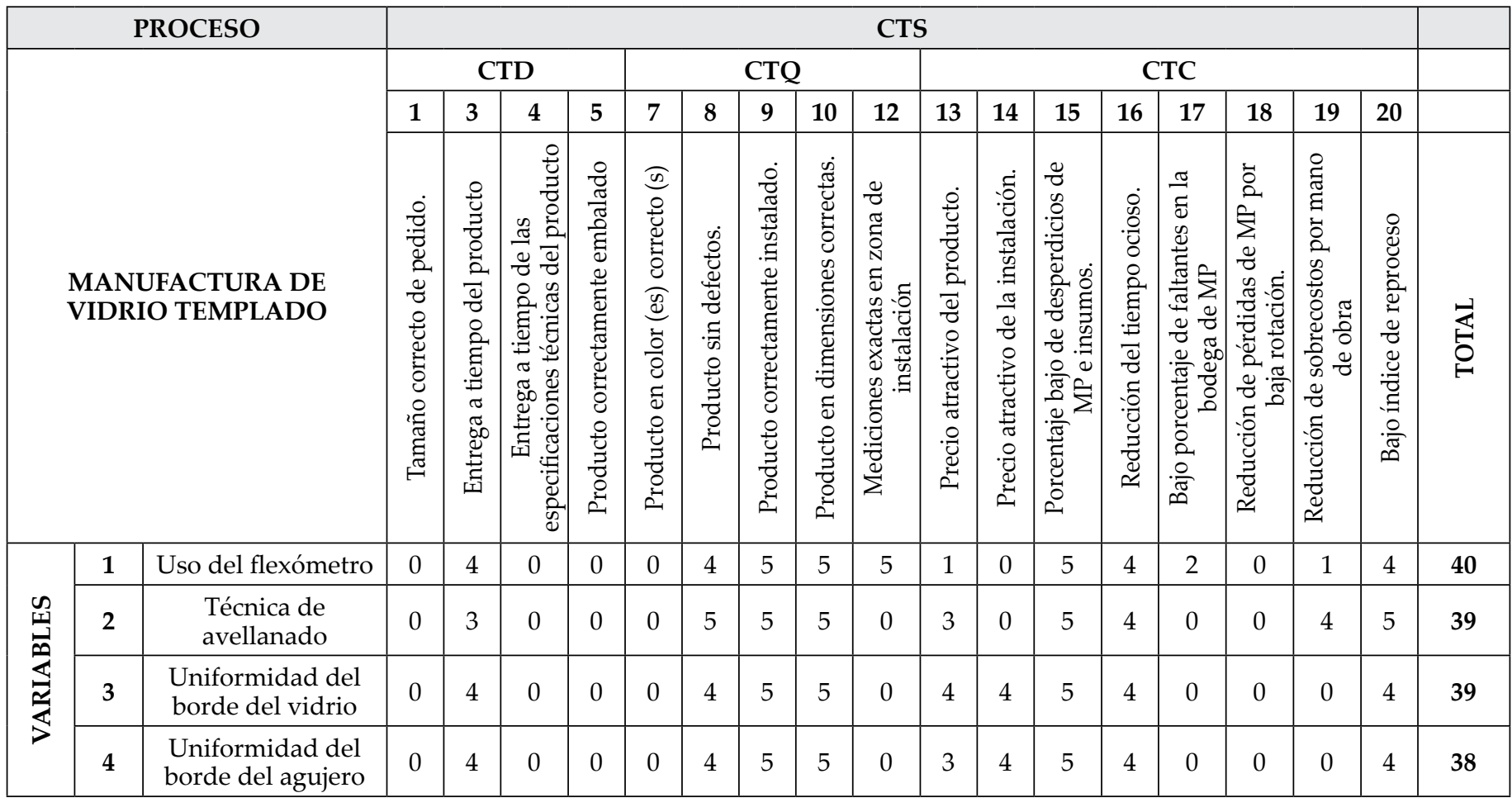

Tabla 7. Modos de falla críticos en la línea de producción de vidrio templado.

Table 7. Critical failure modes in the production line of tempered glass.

\begin{tabular}{|c|c|}
\hline MODO DE FALLA CRÍTICO & RPN \\
\hline Transporte inadecuado del vidrio pulido & 360 \\
\hline Técnica inadecuada de volteo & 360 \\
\hline Descargue incorrecto del vidrio pulido & 360 \\
\hline Técnica inadecuada de avellanado & 360 \\
\hline Error en la ubicación del flexómetro durante la medición & 320 \\
\hline Utilización de la broca incorrecta & 320 \\
\hline Perforadora descalibrada & 315 \\
\hline
\end{tabular}

Tabla 8. Métricos según efecto de falla.

Table 8. KPI for failure effect.

\begin{tabular}{|c|c|c|}
\hline Efecto de falla & Métrico & Frecuencia de medición \\
\hline $\begin{array}{c}\text { Vidrios con dimensiones incorrectas } \\
\text { (reducción/aumento) }\end{array}$ & $\begin{array}{r}\text { Diferencias entre mediciones reales de longitud-ancho y el } \\
\text { patrón establecido }\end{array}$ & $\begin{array}{r}\text { Número de módulos de vidrio quebrados y su causa de quiebre } \\
\text { Rotura/Quiebre }\end{array}$ \\
\hline $\begin{array}{c}\text { Agujero con diámetro fuera de } \\
\text { especificaciones }\end{array}$ & $\begin{array}{r}\text { Diferencias entre mediciones reales del diámetro del agujero y } \\
\text { Borde de agujero no uniforme }\end{array}$ & $\begin{array}{r}\text { Número de módulos de vidrio } \\
\text { reprocesados por borde de agujero no uniforme. }\end{array}$ \\
\hline Corrimiento del agujero & $\begin{array}{r}\text { Diferencias entre mediciones reales del corrimiento del } \\
\text { agujero y el patrón establecido }\end{array}$ & \multirow{2}{*}{ Cada hora } \\
\hline Escarcha en el agujero & $\begin{array}{c}\text { Número de módulos de vidrio reprocesados por escarcha y } \\
\text { número de módulos de vidrio no conformes por escarcha. }\end{array}$ & \\
\hline
\end{tabular}


Tabla 9. Actividades de inspección para puntos de inspección y control.

Table 9. Inspection activities for inspection and control points.

\begin{tabular}{|c|c|}
\hline $\begin{array}{l}\text { PUESTO DE } \\
\text { INSPECCIÓN }\end{array}$ & ACTIVIDADES DE INSPECCIÓN \\
\hline Pulido rectilíneo & $\begin{array}{l}\text { Medición de longitudes y anchos de los } \\
\text { módulos de vidrio. }\end{array}$ \\
\hline \multirow{4}{*}{$\begin{array}{l}\text { Perforado y } \\
\text { boqueteado }\end{array}$} & Medición del diámetro del agujero \\
\hline & $\begin{array}{l}\text { Medición del corrimiento del agujero con } \\
\text { respecto al patrón de medida }\end{array}$ \\
\hline & $\begin{array}{l}\text { Inspección táctil para comprobar } \\
\text { uniformidad del borde del agujero }\end{array}$ \\
\hline & $\begin{array}{l}\text { Inspección visual en búsqueda de } \\
\text { escarchas. }\end{array}$ \\
\hline
\end{tabular}

\section{CONCLUSIONES}

- Las organizaciones deben buscar constantemente distintos métodos para la mejora de la calidad de sus procesos y productos e impactar por ende, el nivel de satisfacción de sus clientes e ingresos. MICEPS es una metodología que responde a estas necesidades de tal manera que los críticos de satisfacción del cliente son traducidos en puntos de inspección y control que garantizan su cumplimiento.

- Apropiadamente implementado, MICEPS genera beneficios en términos de aumento de eficiencia operacional, reducción de costos de no calidad y disminución en los retrasos presentados en la entrega del producto, detección temprana de anomalías en el proceso y reducción de productos defectuosos.

- El marco presentado en este artículo permite la efectiva aplicación de la metodología en cualquier compañía de manufactura, otorgando una poderosa técnica para el monitoreo, análisis y mejora del comportamiento de los procesos a través del uso de métodos estadísticos y de Gestión de Calidad. En algunas organizaciones, sin embargo, podría ser posible que el enfoque de la metodología necesite de algunas adaptaciones.

\section{REFERENCIAS}

[1] R.B. Coronado, J. Antony, "Critical success factors for the successful implementation of six sigma projects in organisations", The TQM Magazine, 14, (2), 92-99, 2002.

[2] G. Box, T. Kramer, "Statistical Process Monitoring and Feedback Adjustment, A Discussion", Technometrics, 34, (1), 251-267, 2012.

[3] J. Maleyeff, F.C. Kaminsky, "Six sigma and introductory statistics education," Education + Training, 44 (2), 8289, 2002.

[4] M. Abreu, R. Cañedo, "Gerencia Total de la calidad en las organizaciones", Acimed, 6, (2), 79 - 92, 1998.

[5] R.J.M. Does, W.A. Schippers, A. Trip, "A framework for implementation of statistical process control", International Journal of Quality Science, 2 (3), 181-198, 1997.

[6] A. Kumar, J. Motwani, "Doing it right the second time", Industrial Management \& Data Systems, 96 (6), 114-19, 1996.

[7] J. Antony, T. Taner, "A conceptual framework for the effective implementation of statistical process control", Business Process Management Journal, 9 (4), 473-489, 2003.

[8] E. Arnheiter, J. Maleyeff, "The Integration of Six Sigma and Lean Management", TQM Magazine, 17 (1), 5 - 18, 2005.

[9] M. Behbahani, A. Saghaee, R. Noorossana, "A case-based reasoning system development for statistical process control: Case representation and retrieval", Computers and Industrial Engineering, 63 (4), 1107 - 1117, 2012.

[10] M. Kulahci, "Multivariate Statistical Process Control”, in Proc. Farm Animal and Food Quality Imaging 2013, Lyngby, 2013, pp. 29.

[11] H. Gutierrez, R. de la Vara, "Análisis de Modo y efecto de las fallas (AMEF)" in Control Estadístico de Calidad y Seis Sigma, $2^{\text {nd }}$ ed. México D.F., México, McGraw Hill, 2009, pp. $406-416$. 